\title{
Ex vivo supplementation with nicotinic acid enhances cellular poly(ADP-ribosyl)ation and improves cell viability in human peripheral blood mononuclear cells
}

\author{
Kathrin Weidele ${ }^{1}$, Andrea Kunzmann ${ }^{1}$, Maike Schmitz, Sascha Beneke, Alexander Bürkle* \\ Molecular Toxicology Group, Department of Biology, University of Konstanz, Konstanz, Germany
}

\begin{abstract}
A B S T R A C T
Poly(ADP-ribosyl)ation is a posttranslational modification of proteins, which is mainly catalyzed by poly(ADP-ribose) polymerase- 1 (PARP-1) by using $\mathrm{NAD}^{+}$as substrate and is directly triggered by DNA strand breaks. Under mild genotoxic stress poly(ADP-ribose) (PAR) formation plays an important role in DNA repair whereas severe genotoxic stress and the ensuing overactivation of PARP-1 induce cellular $\mathrm{NAD}^{+}$depletion, energy failure and ultimately cell death. We are interested in studying the consequences of moderately enhanced enzymatic activity under conditions of DNA damage. Here we chose supplementation of cells with the NAD precursor nicotinic acid (NA) as a strategy. In order to reliably assess PAR accumulation in living cells we first developed a novel, sensitive flow-cytometric method for the rapid analysis of poly(ADP-ribose) accumulation (RAPARA). Our data showed that ex vivo supplementation of human peripheral blood mononuclear cells (PBMC) with low concentrations of NA significantly raised cellular $\mathrm{NAD}^{+}$levels by 2.1 -fold. Upon X-irradiation or exposure to hydrogen peroxide or $N$-methyl- $N^{\prime}$-nitro- $N$-nitrosoguanidine, PAR accumulation was significantly increased and sustained in NA-supplemented cells. Furthermore. NA-supplemented PBMC displayed significantly higher cell viability due to a lower rate of necrotic cell death. In summary, ex vivo supplementation of human PBMC with NA increases cellular NAD ${ }^{+}$levels, boosts the cellular poly(ADP-ribosyl)ation response to genotoxic treatment, and protects from DNA-damage-induced cell death.
\end{abstract}

\section{Introduction}

Poly(ADP-ribosyl)ation is a reversible posttranslational modification of cellular proteins, which is greatly induced after DNA damage [1]. The reaction is catalyzed by poly(ADP-ribose) polymerases (PARPs), a family of enzymes encoded by 17 different genes in the human genome [2,3]. PARP-1 is the best studied family member and is responsible for about $90 \%$ of cellular poly(ADPribose)(PAR) formation after genotoxic stress [4]. PAR formation has been implicated in several cellular processes including DNA repair [5], transcription, chromatin remodeling, and maintenance of genomic stability [6]. After infliction of DNA damage, PARP-1 binds via its zinc finger motifs to DNA single and double strand breaks [7] and synthesizes long polymers of up to 200 units of ADP-ribose on different target proteins, mainly PARP-1 itself, using $\mathrm{NAD}^{+}$as

\footnotetext{
* Corresponding author at: Molecular Toxicology. Department of Biology, Box X911, University of Konstanz, D-78457 Konstanz, Germany. Tel.: +49 7531884035 fax: +497531884033 .

E-mail address: alexander.buerkle@uni-konstanz.de (A. Bürkle).

1 Equal contributors.
}

substrate [8]. The negatively charged polymers have been shown to recruit different DNA repair proteins of the base excision repair (BER) pathway like XRCC I, DNA polymerase $\beta$ and DNA ligase III $[9,10]$. Probably due to charge repulsion, automodified PARP-1 is released from DNA breaks, allowing repair to proceed [11]. PARP activity is counteracted by the enzyme poly(ADP-ribose) glycohydrolase (PARG), of which several splice variants are expressed. PARG possesses exonucleolytic as well as endonucleolytic degrading activity against PAR $[12,13]$. Thus, the polymer level detectable in live cells represents a steady state of anabolic and catabolic activity. However, excessive activation of PARP-1 under circumstances of massive DNA damage induces (i) the depletion of the cellular $\mathrm{NAD}^{+}$ pool, resulting in energy failure concomitant with necrotic cell death [14], or (ii) PAR-dependent release of apoptosis inducing factor (AIF) from mitochondria, thus triggering a caspase-independent form of apoptosis [15]. In mammalian cells, $\mathrm{NAD}^{+}$can be synthesized de novo from tryptophan and in the so-called salvage pathway either from nicotinic acid (NA) or nicotinamide, collectively termed niacin or vitamin B3 [16], or from nicotinamide riboside [17]. Cellular supplementation with NA increases the $\mathrm{NAD}^{+}$pool, thus preventing the extensive loss of $\mathrm{NAD}^{+}[18]$. Cells growing in medium without nicotinamide have much lower $\mathrm{NAD}^{+}$content and as a consequence, 
DNA repair is negatively affected when such cells are exposed to genotoxic agents [19]. In vivo studies on the influence of niacin deficiency [20-22] revealed a decrease in $\mathrm{NAD}^{+}$levels in cells of different tissues accompanied with an impaired PAR content under these conditions. On the other hand, additional intake of NA in vivo increases bone marrow cell $\mathrm{NAD}^{+}$levels as well as poly(ADPribosyl)ation [22]. However, those studies measured PAR content several hours after the treatment with alkylating agents, when cellular response to genotoxic agent could already have influenced other processes. Therefore, it was not defined when and to what extent PARP-1 has been activated initially, as the DNA damage was caused over a longer period of time. But it is important to know if NA supplementation, either in the short term or in the long term, has an impact on the immediate response to DNA damage concerning the duration or intensity of PAR production, as PARP-1 (over)activation is relevant in the pathophysiology of inflammatory or neurodegenerative diseases. The modulation of $\mathrm{NAD}^{+} / \mathrm{PAR}$ metabolism by pharmacological NA supplementation could be an alternative application for pharmacological intervention by altering the activity of PARP-1 and preserving cellular NAD ${ }^{+}$levels to reduce inflammatory responses. To gain a more detailed insight into the sequence of events leading to DNA damage-driven poly(ADP-ribosyl)ation, a higher time resolution and improved detection method is needed. To our knowledge, no study has been published that shows direct consequences of NA supplementation on the duration and intensity of the poly(ADP-ribosyl)ation response to genotoxic treatment and to survival in normal human cells.

Therefore, the goal of our study was to determine the short-term effect of NA supplementation in freshly isolated human peripheral blood mononuclear cells (PBMC) exposed to DNA damaging treatment ex vivo. We addressed the question if increased availability of $\mathrm{NAD}^{+}$can lead to an enhanced cellular poly(ADPribosyl)ation response to DNA damage and, as a consequence, to improved cell viability as a long-term effect. To investigate PAR formation in intact cells, a new flow cytometric assay for the detection of cellular PAR in human PBMC had to be established. Using this method termed RAPARA (rapid analysis of polylaDPribosel accumulation), we could show that supplementation with NA indeed leads to a increased and sustained poly(ADP-ribosyl)ation after X-irradiation, hydrogen peroxide or MNNG treatment in PBMC, further demonstrating that the cellular $\mathrm{NAD}^{+}$content is a critical determinant for the level of PAR formation. In addition, the supplemented cells showed a higher viability after DNA damage.

\section{Materials and methods}

\subsection{Cells}

Blood samples (collected in citrate S-Monovettes from Sarstedt, Nümbrecht, Germany) were obtained from healthy male or female donors aged 20-45. The research was conducted in accordance with the Declaration of Helsinki, and ethical clearance was obtained from the University of Konstanz Ethics Committee. For all experiments freshly isolated PBMC were used. These were cultured between $5 \mathrm{~h}$ and 1 day in RPMI containing $10 \% \mathrm{FCS}, 100 \mathrm{U} /$ $\mathrm{ml}$ penicillin and $100 \mu \mathrm{g} / \mathrm{ml}$ streptomycin at $37^{\circ} \mathrm{C}$ and $5 \% \mathrm{CO}_{2}$ in a humidified atmosphere. Medium and supplements were purchased from Invitrogen (Darmstadt, Germany), FCS from Biochrom (Berlin, Germany).

\subsection{Isolation of $P B M C$}

Ten $\mathrm{ml}$ freshly obtained peripheral venous blood was diluted with $20 \mathrm{ml}$ phosphate buffered saline(PBS; $137 \mathrm{mM} \mathrm{NaCl}$ [Carl Roth,

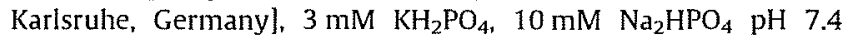
[Sigma-Aldrich, Munich, Germany]), placed on top of $15 \mathrm{ml}$ Biocoll
(Biochrom) in a 50-ml conical tube and centrifuged without brake at $800 \times \mathrm{g}$ for $10 \mathrm{~min}$ at room temperature. The PBMC recovered were washed twice with PBS and used for the experiments or kept in culture [23]

\subsection{Supplementation of PBMC with nicotinic acid}

For each experiment, one half of the freshly isolated PBMC were supplemented with $15 \mu \mathrm{M}$ NA (Merck, Darmstadt, Germany) for at least $5 \mathrm{~h}$. The other half was incubated in the culture medium without NA supplementation as a control.

\subsection{PARP inhibition}

Cells were incubated with PARP inhibitor PJ-34 (Enzo Life Sciences, Lörrach, Germany) for at least $5 \mathrm{~h}$ at final concentrations of $0.03,0.3$ or $3 \mu \mathrm{M}$ before and during genotoxic exposure.

\subsection{Determination of PARP-1 activity in intact PBMC by flow cytometry}

Aliquots of $5 \times 10^{5}-1 \times 10^{6}$ cells resuspended in $80 \mu \mathrm{l}$ PBS were placed into each well of a 96-well v-bottom plate (Sarstedt). Cells were X-irradiated (RT 100, Müller, Hamburg, Germany) on ice at doses between 0 and $25 \mathrm{~Gy}$, incubated at $37^{\circ} \mathrm{C}$ for the time periods indicated and subsequently fixed and permeabilized with $180 \mu \mathrm{l}$ methanol (Sigma-Aldrich)/acetic acid $(3+1)$ (Carl Roth) for 5 min at room temperature. Altematively, cells were treated with hydrogen peroxide (Merck) at final concentrations as indicated for $3 \mathrm{~min}$ at $37^{\circ} \mathrm{C}$ or with MNNG (Sigma-Aldrich) for $10 \mathrm{~min}$ at room temperature to induce PAR synthesis. Both chemicals were removed by centrifugation, $200 \times \mathrm{g}$ for $3 \mathrm{~min}$, and a further washing step with PBS. Cell pellets were resuspended with $80 \mu \mathrm{l}$ PBS and subsequently fixed as described.

Cells were centrifuged at $1180 \times \mathrm{g}$, washed twice with FACS buffer (PBS; $0.5 \%$ FCS; $2 \mathrm{mM} \mathrm{NaN}_{3}$ [Merck]) and incubated with $100 \mu \mathrm{l}$ primary antibody $10 \mathrm{H}(5 \mu \mathrm{g} / \mathrm{ml})$ recognizing PAR [24] for $1 \mathrm{~h}$ at room temperature. Cells were centrifuged at $1180 \times \mathrm{g}$ and washed twice with $200 \mu$ l FACS buffer before incubated with the Alexa488-labeled goat-anti-mouse secondary antibody (Invitrogen) for $1 \mathrm{~h}$ at room temperature. Finally, cells were centrifuged at $1180 \times g$, washed twice with FACS buffer and kept on ice until flow cytometric analysis was performed (FACS Calibur II or LSR II, Becton Dickinson, Heidelberg, Germany). A total of 10,000 events for each sample were acquired and analyzed using FlowJo V7 software (Tree Star Inc., Ashland OR, USA).

\subsection{Assessment of cell viability by flow cytometry}

Cell viability was determined using Annexin $V$ staining (Enzo Life Sciences) according to the manufacturer's protocol. Cells were irradiated with 0 or 5 or 25 Gy. After $24 \mathrm{~h}$, cells were washed with PBS and $10^{6}$ cells were resuspended $1 \mathrm{ml}$ binding buffer $(140 \mathrm{mM}$ $\mathrm{NaCl}$ [Carl Roth], $10 \mathrm{mM}$ HEPES and $2.5 \mathrm{mM} \mathrm{CaCl}_{2}$ [both from SigmaAldrich], pH 7.4). For each treatment $100 \mu \mathrm{l}$ of the cell suspension was mixed with $5 \mu$ l annexin $V$ and propidium iodide (final concentration $1 \mu \mathrm{g} / \mathrm{ml}$; Sigma-Aldrich) and incubated in the dark at room temperature. After $15 \mathrm{~min}, 400 \mu \mathrm{l}$ of binding buffer was added to each sample and flow cytometric analysis was performed (FACS Calibur II or LSR II, BD). A total of 10,000 events for each sample were acquired and analyzed using FlowJo V7 software (Tree Star).

\subsection{Quantification of cellular $N A D^{+}$content}

The cellular $\mathrm{NAD}^{+}$content was determined using a modified $\mathrm{NAD}^{+}$cycling assay [25]. For each treatment $3-5 \times 10^{6}$ cells were 
(A)

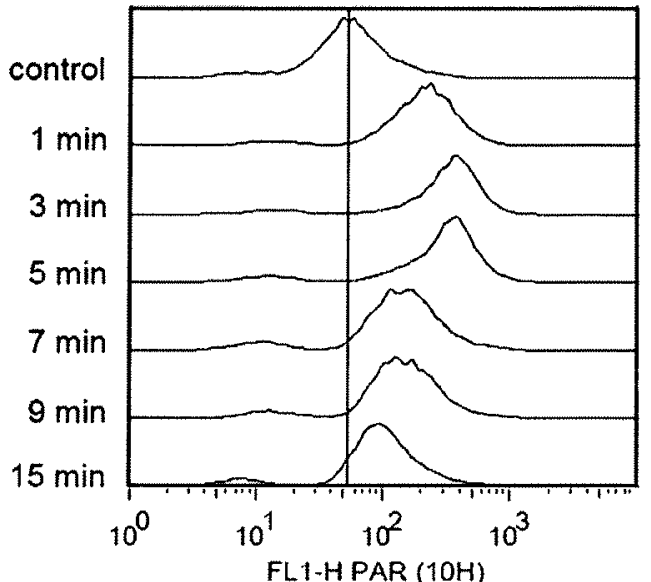

Fluorescence intensity

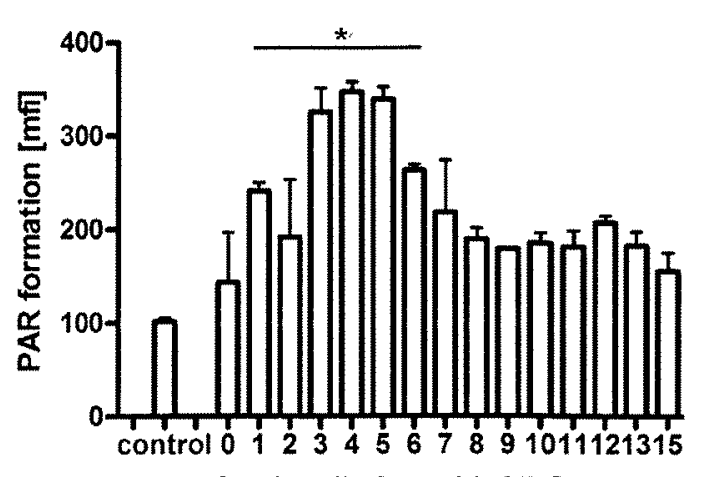

Time after irradiation with 25 Gy [min]

(B)
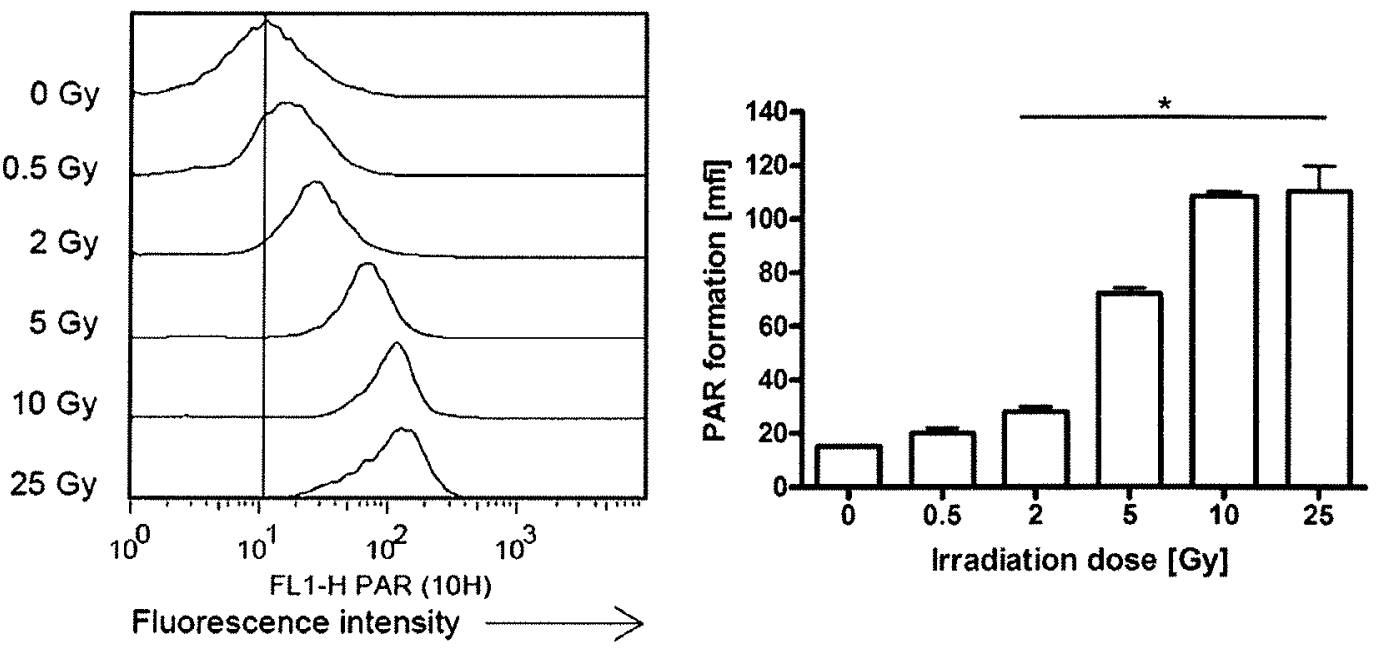

Fig. 1. Evaluation of RAPARA for the detection of poly(ADP-ribosyl)ation in intact PBMC in response to X-irradiation. Single-parameter histograms of poly(ADP-ribose)associated mean fluorescence intensities and quantitative evaluation are displayed. (A) Cells were treated with $25 \mathrm{~Gy}$ and incubated at $37^{\circ} \mathrm{C}$ for different time periods. PAR level is increased up to 4 min after damage; thereafter the PAR-degrading activity of PARG exceeds PAR synthesis. A significant increase is detectable from 1 min on $\left({ }^{*} p<0.05\right.$ repeated measures ANOVA). Control represents non-irfadiated cells. (B) Cells were treated with different doses of X-irradiation and incubated at $37{ }^{\circ} \mathrm{C}$ for 4 min. There is already a noticeable increase in polymer formation after an irradiation dose of $0.5 \mathrm{~Gy}$ and a significant increase after a dose of 2 Gy ${ }^{*}{ }^{*} p<0.05$ repeated measures ANOVA), thus highlighting the high sensitivity of the new assay. Histograms are representative of one out of 3 measurements of one donor. Each bar represents triplicate analysis of PBMC from one donor and is expressed as mean \pm S.D.

resuspended in $500 \mu \mathrm{l}$ PBS, $\mathrm{X}$-irradiated as indicated (0-25 Gy) and incubated at $37^{\circ} \mathrm{C}$ for different time periods $(0-15 \mathrm{~min})$. If treated with $\mathrm{H}_{2} \mathrm{O}_{2}$ or $M N N G$, the cells were subsequently centrifuged, washed and resuspended in $500 \mu \mathrm{l}$ PBS. Thereafter, cells were precipitated with $0.5 \mathrm{M} \mathrm{HClO}_{4}$ on ice. After $15 \mathrm{~min}$ samples were centrifuged at $1500 \times \mathrm{g}$ for $10 \mathrm{~min}$ and the supernatant was combined with $350 \mu \mathrm{l}$ of $1 \mathrm{M} \mathrm{KOH}, 0.33 \mathrm{M}$ $\mathrm{K}_{2} \mathrm{HPO}_{4}, 0.33 \mathrm{M} \mathrm{KH}_{2} \mathrm{PO}_{4}$ followed by incubation on ice for $10 \mathrm{~min}$. All chemicals were from Sigma-Aldrich. Cells were centrifuged at $1500 \times \mathrm{g}$ for $10 \mathrm{~min}$ and the supernatant was frozen at $-20^{\circ} \mathrm{C}$ before $\mathrm{NAD}^{+}$determination by an enzymatic cycling assay [25].

\subsection{Statistical analysis}

All statistical analyses were done with Prism or InStat (GraphPad Inc., La Jolla, CA, USA). If two groups were compared, a two-tailed paired $t$-test was performed. For the comparison of more than two groups, analysis of variance (ANOVA) was used. A pvalue $<0.05$ was considered significant.

\section{Results}

3.1. Establishing a new method to detect PARP activity and determination of cellular PAR contents in intact cells

To determine cellular PAR accumulation in intact cells, a new flow-cytometry based assay termed RAPARA (rapid analysis of poly[ADP-ribose] accumulation) was established.

Using this new assay, it is possible to closely monitor the timedependent (Fig. 1A) as well as dose-dependent (Fig. 1B) cellular polymer formation after irradiation with high resolution. Upon irradiation with $25 \mathrm{~Gy}$ we detected significant induction of PAR formation already after 1 min of incubation at $37^{\circ} \mathrm{C}(p<0.05)$ (Fig. 1A). At the time of highest PAR accumulation (i.e., between 3 and $5 \mathrm{~min}$ after irradiation), we were able to measure induction of PARP activity already after a dose of 0.5 Gy. PARP activity was significant different from control when irradiated with $2 \mathrm{~Gy}$ $(p<0.05)$ or higher doses (Fig. 1B). The results confirm that poly(ADP-ribosyl)ation is a very fast response to DNA damage in 
PBMC, as there is a detectable increase even after $1 \mathrm{~min}$ of incubation, and maximum PAR accumulation can be observed already after 3-5 min in this cell type. For comparison, Supplementary Fig. S1 shows a western blot analysis of whole cell lysates of cells treated with X-irradiation. With this standard technique PAR formation is clearly detectable after high dose irradiation, but compared to the RAPARA assay lacks sensitivity in the low dose range.

\subsection{Effect of nicotinic acid supplementation on cellular $N A D^{+}$level}

NA is a precursor of the PARP substrate NAD'. Supplementation with NA was done immediately, if not indicated otherwise, after $P B M C$ isolation in culture medium for $5 \mathrm{~h}$, in order to ensure efficient uptake and metabolism of NA. To determine which concentrations of supplemental NA can induce increased biosynthesis of $\mathrm{NAD}^{+}$and therefore boost the $\mathrm{NAD}^{+}$pool, a range of concentrations were tested. Addition of increasing concentrations of NA to routine culture medium, which usually contains about $8.2 \mu \mathrm{M} N A$, elevated total cellular $\mathrm{NAD}^{+}$levels in PBMCs in a concentration-dependent manner (Supplementary Fig. S2). Significant increase was observed above $5 \mu \mathrm{M}$. Robust and effective augmentation was achieved with a concentration of $15 \mu \mathrm{M} N A$ (Table 1), which was therefore used for all subsequent experiments. An increase in $\mathrm{NAD}^{+}$level was recorded in the cells from 13/13 donors, regardless if freshly isolated or 1 day cultured cells (labeled with asterisk) were supplemented. Amongst the donors, there was no significant gender effect detectable with regards to basal NAD levels or the effect of supplementation. Furthermore, supplementation had no effect on cell volume ( $200 \mu \mathrm{m}^{3}$ on average).

\subsection{Effect of nicotinic acid supplementation on PAR accumulation}

To induce cellular poly(ADP-ribosyl)ation, PBMC were $\mathrm{X}$-irradiated with doses from 1 to $25 \mathrm{~Gy}$ and further incubated for different time periods. PAR accumulation was determined by RAPARA. For the dose-response experiments (Fig. 2A and B), cells were incubated for $3 \mathrm{~min}$ after irradiation to measure the peak of cellular poly(ADP-ribosyl)ation, as was determined in Fig. 1A. Our data revealed that the NA- dependent increase of $\mathrm{NAD}^{+}$levels also increased cellular PAR formation in PBMC, which was significant when treated with doses of $5 \mathrm{~Gy}$ and above (Fig. $2 \mathrm{~A}$ ). The dosedependent increase in poly(ADP-ribosyl)ation was enhanced when cells were supplemented with NA, with the strongest effect being

\section{Table 1}

Inter-individual variation in cellular $\mathrm{NAD}^{*}$ concentration in human PBMC supplemented or not with NA. $m$, male; f, female; asterisks indicate that cells were cultured for 1 day before NA supplementation. Two-tailed paired $t$-test was used for statistical evaluation.

\begin{tabular}{|c|c|c|c|c|}
\hline Donor & Gender & $\begin{array}{l}\text { Intracellular NAD } \\
\text { concentration } \\
{[\mu \mathrm{M} \mid \text { without NA }} \\
\text { supplementation }\end{array}$ & $\begin{array}{l}\text { Intracellular NAD } \\
\text { concentration } \\
\text { | } \mu \mathrm{M} \mid \text { with NA } \\
\text { supplementation }\end{array}$ & $\begin{array}{l}\text { Fold } \\
\text { increase }\end{array}$ \\
\hline 01 & $\mathrm{~m}$ & 194.6 & 295.5 & 1.5 \\
\hline 02 & $\mathrm{~m}$ & 170.0 & 307.3 & 1.8 \\
\hline 03 & $\mathrm{~m}$ & 174.9 & 382.5 & 2.1 \\
\hline 04 & $f$ & 181.8 & 380.4 & 2.1 \\
\hline 05 & $\mathrm{f}$ & 190.0 & 489.0 & 2.6 \\
\hline $06^{\circ}$ & f & 215.1 & 490.7 & 2.2 \\
\hline $07^{*}$ & f & 239.5 & 579.1 & 2.4 \\
\hline $08^{*}$ & $\mathrm{f}$ & 134.9 & 329.1 & 2.4 \\
\hline $09^{*}$ & $\mathrm{f}$ & 230.9 & 560.3 & 2.4 \\
\hline 10 & $\mathrm{f}$ & 202.4 & 390.7 & 1.9 \\
\hline $11^{*}$ & $\mathrm{~m}$ & 249.3 & 383.1 & 1.5 \\
\hline 12 & $\mathrm{~m}$ & 173.1 & 370.3 & 2.1 \\
\hline \multirow[t]{2}{*}{13} & f & 202.6 & 487.6 & 2.4 \\
\hline & & Mean \pm S.D. & & $2.1 \pm 0.3$ \\
\hline
\end{tabular}

apparent at the highest dose tested (25 Gy). In contrast, there was no change in the basal activity of PARP- 1 by NA supplementation (Fig. 2A). Concomitant with the increased poly(ADP-ribosyl)ation observed at higher irradiation doses, there was a decrease of cellular NAD ${ }^{+}$level, which was mitigated in cells supplemented with NA (Fig. 2B). The results from the time-course experiments are perfectly in line with this observation (Fig. 2C and D).

The response of PARP-1 to DNA damage is time-dependent, showing a very fast maximum within the first minutes, before PAR becomes degraded (Fig. 2C). In PBMC the highest level of PAR can always be detected at the same time point, i.e. $1 \mathrm{~min}$ after irradiation, independent of the available $\mathrm{NAD}^{+}$. Cellular $\mathrm{NAD}^{+}$ levels dropped simultaneously within the first minutes after irradiation (Fig. 2D). The total amount of both, PAR and NAD ${ }^{+}$was much higher at all time points with NA supplementation than without. It is also apparent that the increased PAR level persisted longer in NA-supplemented cells.

Additionally we monitored PAR formation and cellular NAD depletion in response to oxidant or alkylation-induced DNA damage. The DNA-damaging compounds used, $\mathrm{H}_{2} \mathrm{O}_{2}$ and $\mathrm{MNNG}$ led to an increase of polymer formation (Fig. $3 \mathrm{~A}$ and $\mathrm{C}$ ) with significantly higher levels in NA-supplemented cells. The effect on cellular $\mathrm{NAD}^{+}$consumption (Fig. $3 \mathrm{~B}$ and D) was comparable with that in Fig. 2B, where DNA damage was induced by irradiation, showing that $\mathrm{NAD}^{+}$pools decline gradually with increasing PAR formation and independent of the DNA-damaging agent applied.

We used PARP inhibitor PJ-34 in a range of concentrations in order to monitor the impact on PAR formation and NAD ${ }^{+}$depletion (Fig, 4). In response to $25 \mathrm{~Gy} X$-irradiation the cells show concentration-dependent inhibition of PAR formation (Fig. 4A) At the lowest concentration of PJ-34 the PAR level of NAsupplemented cells was still higher compared to control, but further increasing PJ-34 concentration led to a complete block of PAR formation. The drop of $\mathrm{NAD}^{+}$level after irradiation with $25 \mathrm{~Gy}$ was reduced as a function of PJ-34 concentrations (Fig. 4B). Low concentrations of PJ-34 attenuated the consumption of $\mathrm{NAD}^{+}$ partially, whereas PARP inhibition with $3 \mu \mathrm{M} \mathrm{PJ-34}$ totally prevented NAD ${ }^{+}$loss, due to the inability of cells to produce PAR

\subsection{Effect of NA on cell viability}

As the strand break-stimulated PARP-1 activity can consume large amounts of $\mathrm{NAD}^{+}$, it was important to investigate if the increased $\mathrm{NAD}^{+}$concentration and enhanced poly(ADP-ribosyl)ation after NA supplementation is linked with an increase in cell viability after DNA damage. To analyze the impact of NA supplementation on apoptosis and necrosis, supplemented and non-supplemented cells were irradiated with 0 or 5 or $25 \mathrm{~Gy}$ and incubated in culture medium $(-1+N A)$ for $24 \mathrm{~h}$ at $37^{\circ} \mathrm{C}$, followed by annexin $\mathrm{V}$ and propidium iodide staining. Ionizing radiation induced a dose-dependent increase in cell death (Fig. 5A). In undamaged cells, there was no difference in cell viability between NA-supplemented and non-supplemented cells. We calculated the ratio of total and necrotic cell death of non-supplemented or NAsupplemented PBMCs to unirradiated controls (Fig. 5B). Upon irradiation, there was a decrease in cell death rates in NAsupplemented cells by $30 \%$ (4.2-fold induction without NA vs. 3 fold induction with NA). This was mainly the result of a reduced rate of necrosis, which dropped by $45 \%$ after NA supplementation compared to non-supplemented cells. Thus, under conditions of higher PARP- 1 activity and an increased NAD ${ }^{+}$concentration, cells are more resistant to DNA damage.

To test if the observed effect on cell death is a direct consequence of the increased PARP-1 activity or only correlated to it, we inhibited PARP with PJ-34 (Fig. 6). Total cell death after 5 or $25 \mathrm{~Gy}$ irradiation was diminished in NA-supplemented cells in 

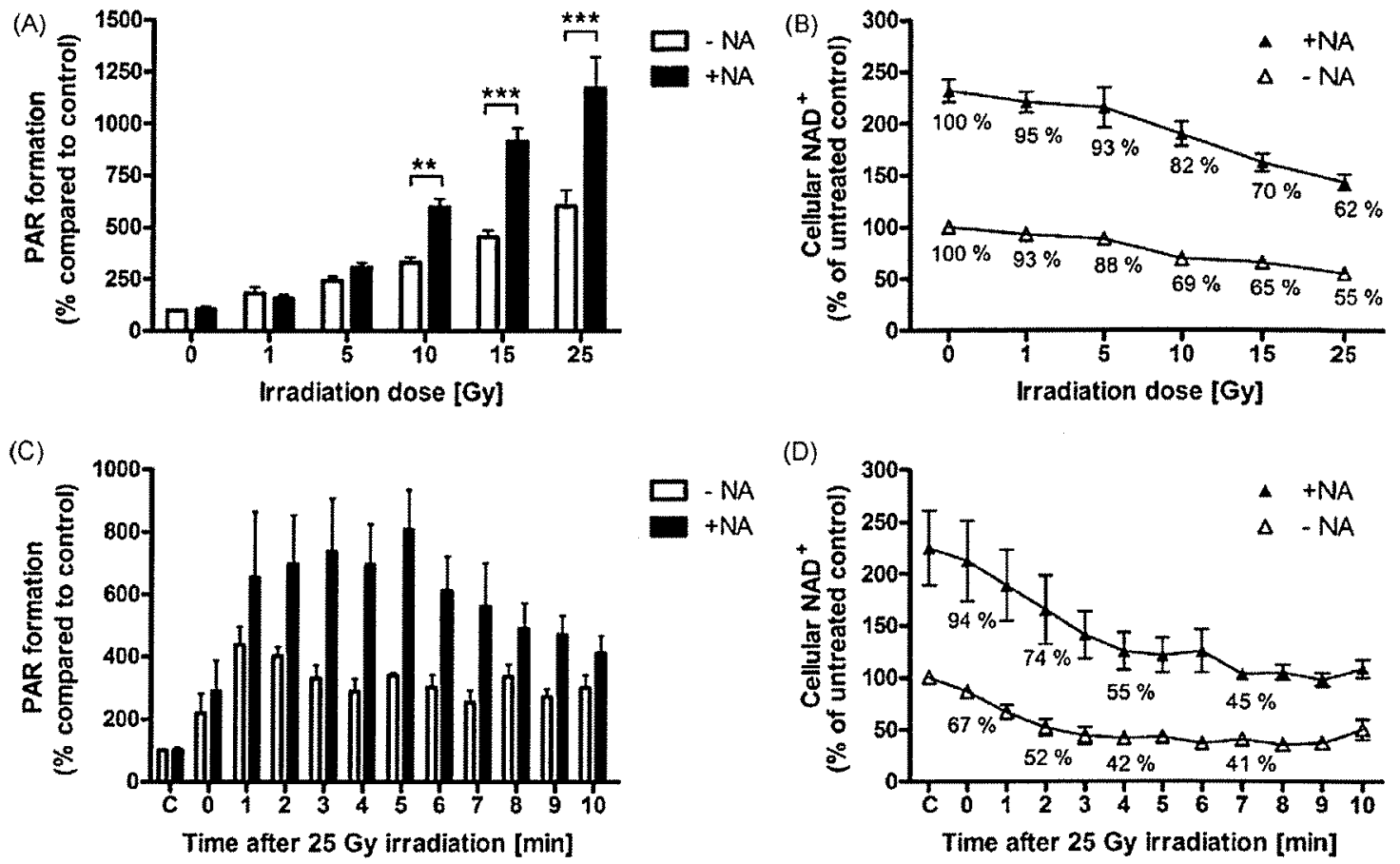

Fig. 2. Effect of NA supplementation on PAR accumulation and the cellular NAD level. Poly(ADP-ribosyl)ation and NAD ${ }^{+}$was measured upon $X$-irradiation of PBCM. The cells were irradiated with doses as indicated and incubated for 3 min at $37^{\circ} \mathrm{C}$ ( $\mathrm{A}$ and $\mathrm{B}$ ) or PBMC were irradiated with $25 \mathrm{Cy}$ and incubated at $37^{\circ} \mathrm{C}$ for different time points as indicated ( $C$ and $D$ ) Fach bar represents the mean of triplicate measurements from three donors, respectively, and is expressed in \% ( \pm SEM). (A) Dose-dependent response of PARP-1 activity in NA-supplemented compared with non-supplemented controls. A significant difference was detected at $10 \mathrm{~Gy}$ or above $\left({ }^{* *} p<0.01\right.$ : ${ }^{* * *} p<0.001$, repeated measures ANOVA). (B) Effect of NA supplementation on the cellular NAD concentration compared with non-supplemented controls after different irradiation doses, (C) Timedependent response of PARP-1 activity in non-supplemented and NA-supplemented cells. (D) Time-dependent effect of NA supplementation on the cellular NAD ${ }^{+}$concentration compared with non-supplemented controls.
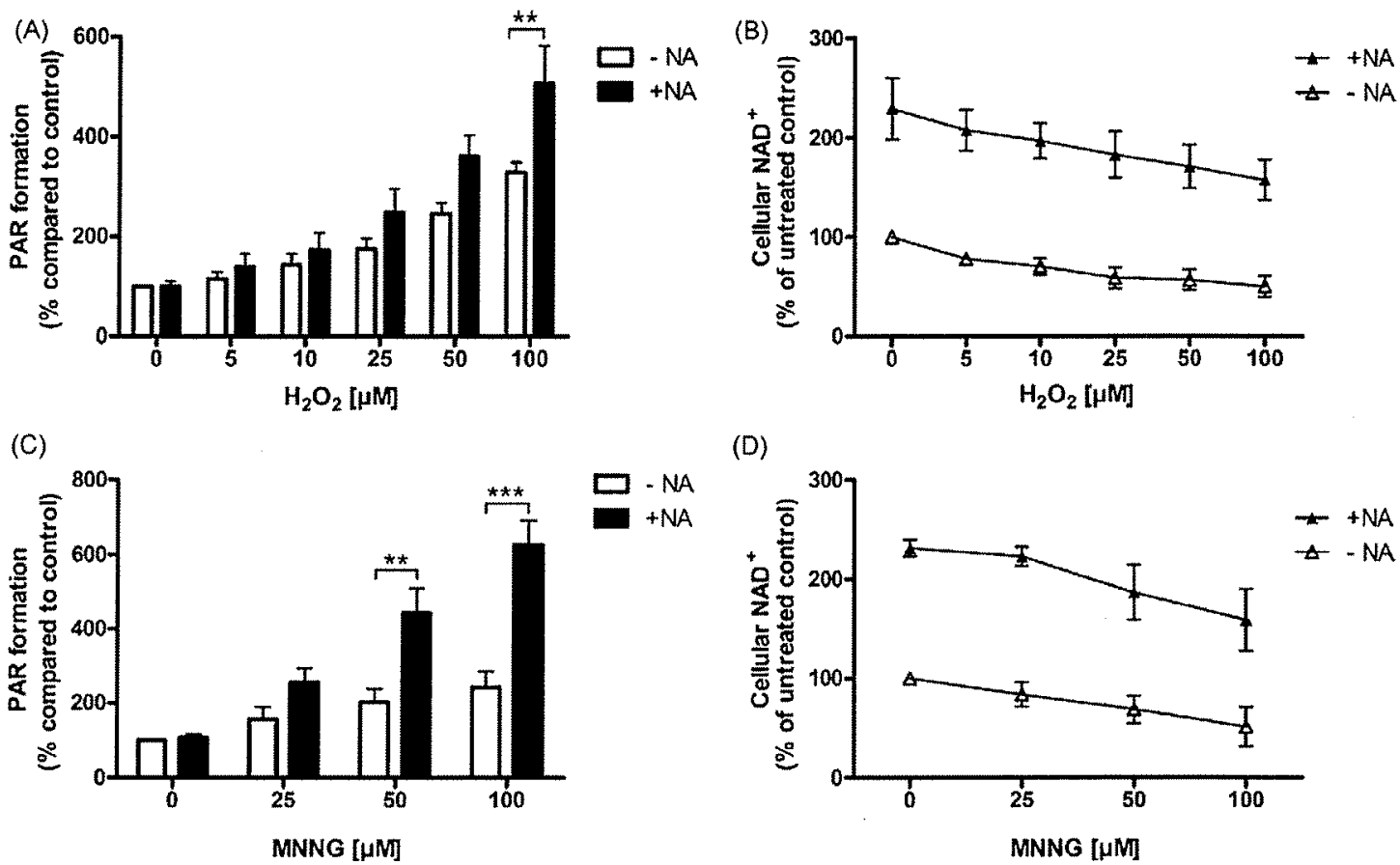

(D)

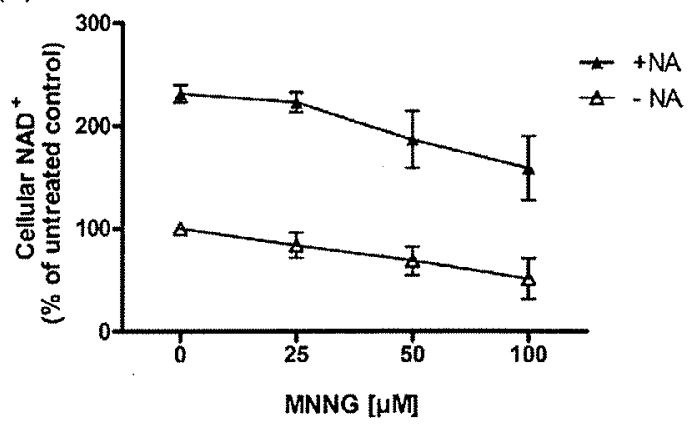

Fig. 3. Effect of NA supplementation on PAR accumulation and the cellular $N A D^{+}$level in response to oxidative stress ( $A$ and $B$ ) or alkylating agents ( $C$ and $D$ ). Each bar represents the mean of triplicate measurements from four donors, respectively, and is expressed as \% ( $\mathrm{SSEM}$ ). ( $\mathrm{A}, \mathrm{C}$ ) PAR levels induced by different concentration of $\mathrm{H}_{2} \mathrm{O}_{2}$ ( $\mathrm{A}$ ) or MNNG (C) were significantly higher for NA-supplemented cells as indicated $\left({ }^{* *} p<0.01 ;{ }^{* * *} p<0.001\right.$, repeated measures ANOVA). (B, D) Cellular NAD ${ }^{*}$ levels decreased in a concentration-dependent manner after treatment with hydrogen peroxide (B) or MNNG (D). 

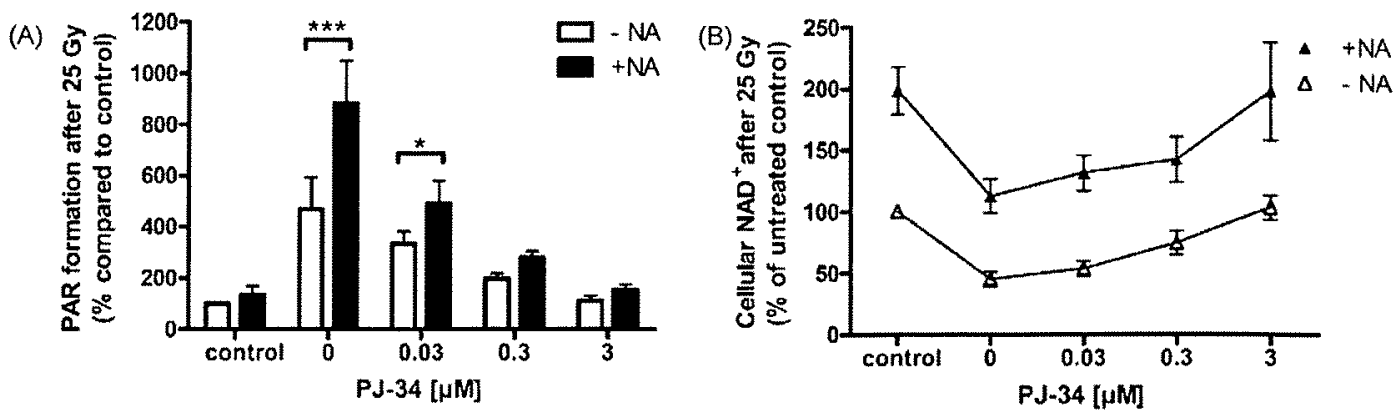

Fig. 4. Effect of PJ-34 on PAR accumulation and NAD levels after X-irradiation. PBMCs were treated with PJ-34 as indicated and assayed for PAR formation and cellular NAD ${ }^{+}$ concentration 3 min after irradiation with $25 \mathrm{~Gy}$. Each bar represents the mean of triplicate measurements from four donors, respectively, and is expressed as \% ( \pm SEM). (A) Impact of increasing concentrations of PJ 34 on PAR levels in non-supplemented and NA-supplemented cells. (B) Impact of increasing concentrations of PJ-34 on cellular NAD ${ }^{+}$levels in NA-supplemented cells compared to non-supplemented controls.

contrast to non-supplemented (Fig. 6A and B). This was independent of the addition of PJ-34. The frequency of necrotic cell death significantly decreased at concentrations of 0.3 and $3 \mu \mathrm{M} \mathrm{PJ}-34$ in NA or non-supplemented PBMC (Fig. 6C and D). By contrast, increasing concentrations of the PARP inhibitor led to an increase in the apoptotic fraction after irradiation with 5 or $25 \mathrm{~Gy}$ (Fig. $6 \mathrm{E}$ and $\mathrm{F}$ ).

\section{Discussion}

In this study we focused on the consequences of elevated $\mathrm{NAD}^{+}$ levels regarding PARP-1 activation after DNA damage induction by ionizing irradiation, $\mathrm{H}_{2} \mathrm{O}_{2}$ or MNNG treatment. Intracellular $\mathrm{NAD}^{+}$ levels of human PBMC were raised by ex vivo supplementation of culture medium with low concentrations $(15 \mu \mathrm{M})$ of $\mathrm{NAD}^{+}$ precursor NA. We found a strong and robust increase in cellular $\mathrm{NAD}^{+}$levels, with the basal NAD ${ }^{+}$status being increased 2.1 -fold on average. This concentration of the precursor is much higher than fasting levels of NA in human blood plasma, which is approximately $0.08 \mu \mathrm{M}[26]$ or barely detectable, because NA is largely converted to $\mathrm{NAD}^{+}$and therefore does not persist in circulating blood.

As non-supplemented cells were kept in culture medium, whose components are designed to maximize cell growth and survival rather than to perfectly model in vivo conditions, controls were also exposed to $\mathrm{NAD}^{+}$precursors due to the routine media supplements [27]. However, this led only to a slight increase of basal $\mathrm{NAD}^{+}$pools after incubation of $5 \mathrm{~h}$ (medium control, Supplementary Fig. S2) or longer (24 h, marked with asterisk, Table 1). Nevertheless, we decided to use the shorter $(5 \mathrm{~h})$ incubation period in order to avoid non-physiological conditions.

We then monitored the immediate impact on PAR formation in response to DNA damage using the newly developed RAPARA. We could track the time and dose-dependent PAR formation after PARP activation with high resolution. Next, we analyzed cell viability, since PARP-1 is implicated in cell death processes by excessive consumption of NAD ${ }^{+}$. We found that cell death in NA-supplemented cells was reduced after irradiation due to effective protection from necrotic cell death.

RAPARA enabled us to assess PAR levels in intact living primary cells under physiological conditions. Using this new approach we can detect PAR levels and the direct impact on PAR formation after addition of nutritional factors like NA, the effect of DNA-damaging agents (Figs, 2 and 3 ) or PARP inhibitors (Fig. 4). It should be noted that there are several types of PARP activity assays available, but most of them can only be used for measurements in permeabilized cells $[28,29]$ or in cell lysates [30]. For example, another flow cytometric PAR assay we have published previously (Kunzmann, 2006) comprises permeabilization of cells and postincubation of the cell "ghosts" with saturating concentrations of exogenous $\mathrm{NAD}^{+}$and an PARP activator oligonucleotide in order to assess total cellular poly(ADP-ribosyl)ation capacity [28]. On the other hand the determination of PARP-1 activity in intact cells, i.e. the PAR detected being produced by the living cell, as a function of its PARP activation status, endogenous $\mathrm{NAD}^{+}$pools and perhaps other factors, was so far restricted to either biochemical extraction of PAR followed by HPLC-based quantitative detection of enzymatic digestion products [31,32], which is sensitive and accurate but requires large cell numbers, or immunofluorescence microscopy [33], which is neither quantitative nor useful for the comparison of large sets of samples.

In contrast, RAPARA is very sensitive as we are able to detect routinely PAR formation even after low-level genotoxic stimuli application, which escape detection by the current gold standard, i.e. western blotting (Fig. S1). A slight increase, though not statistically significant, in PAR levels can be detected by RAPARA after X-irradiation with as little as $0.5 \mathrm{~Gy}$ or $\mathrm{H}_{2} \mathrm{O}_{2}$ addition of $5 \mu \mathrm{M}$ compared to controls. Significantly increased levels were observed after $2 \mathrm{~Gy}$ irradiation or $25 \mu \mathrm{M} \mathrm{H}_{2} \mathrm{O}_{2}$, which was not achieved using the western blot technique (Fig. S1). Furthermore with RAPARA it is possible to determine PARP activity conveniently and with high temporal resolution (Fig. 1A). The apparent difference in the maximal PAR accumulation between Fig. 1A and Fig. 2D can be explained by the inter-individual variability of PAR formation in response to DNA damage [28]. As RAPARA is able to yield results quickly and with high sensitivity, it holds great opportunity for measuring PARP-1 activity in intact PBMC. This is an enormous advantage in studies where PARP-1 activity is, for example, used as a biomarker, as it has already been done in permeabilized PBMC [34].

In the present work we used RAPARA to assess the effect of elevated intracellular $\mathrm{NAD}^{+}$levels on PAR formation after DNA damage induced by genotoxic agents, and we could confirm that the higher availability of $\mathrm{NAD}^{+}$positively affects cellular poly(ADPribosyl)ation, which is in agreement with other studies $[18,35]$. Moreover we showed that the same amount of DNA damage leads to a higher level of poly(ADP-ribosyl)ation in NA-supplemented cells, even after low-dose irradiation when the availability of $\mathrm{NAD}^{+}$ is not limited. We also used hydrogen peroxide and MNNG, two DNA-damaging substances triggering PARP activity, to show that the observed effects concerning NAD ${ }^{+}$and PAR formation after NAsupplementation are independent of the type of DNA damage. In NA-supplemented cells, PAR persists for extended time periods (Fig. 2D). It has been shown in vitro that $\mathrm{NAD}^{+}$concentration can affect length and frequency of branching sites of PAR chains [36]. Thus, the higher NAD ${ }^{*}$ concentration may possibly lead to higher amount of branched polymer, which was reported to be degraded more slowly by PARG [37]. Also, the increased availability of $\mathrm{NAD}^{+}$ 

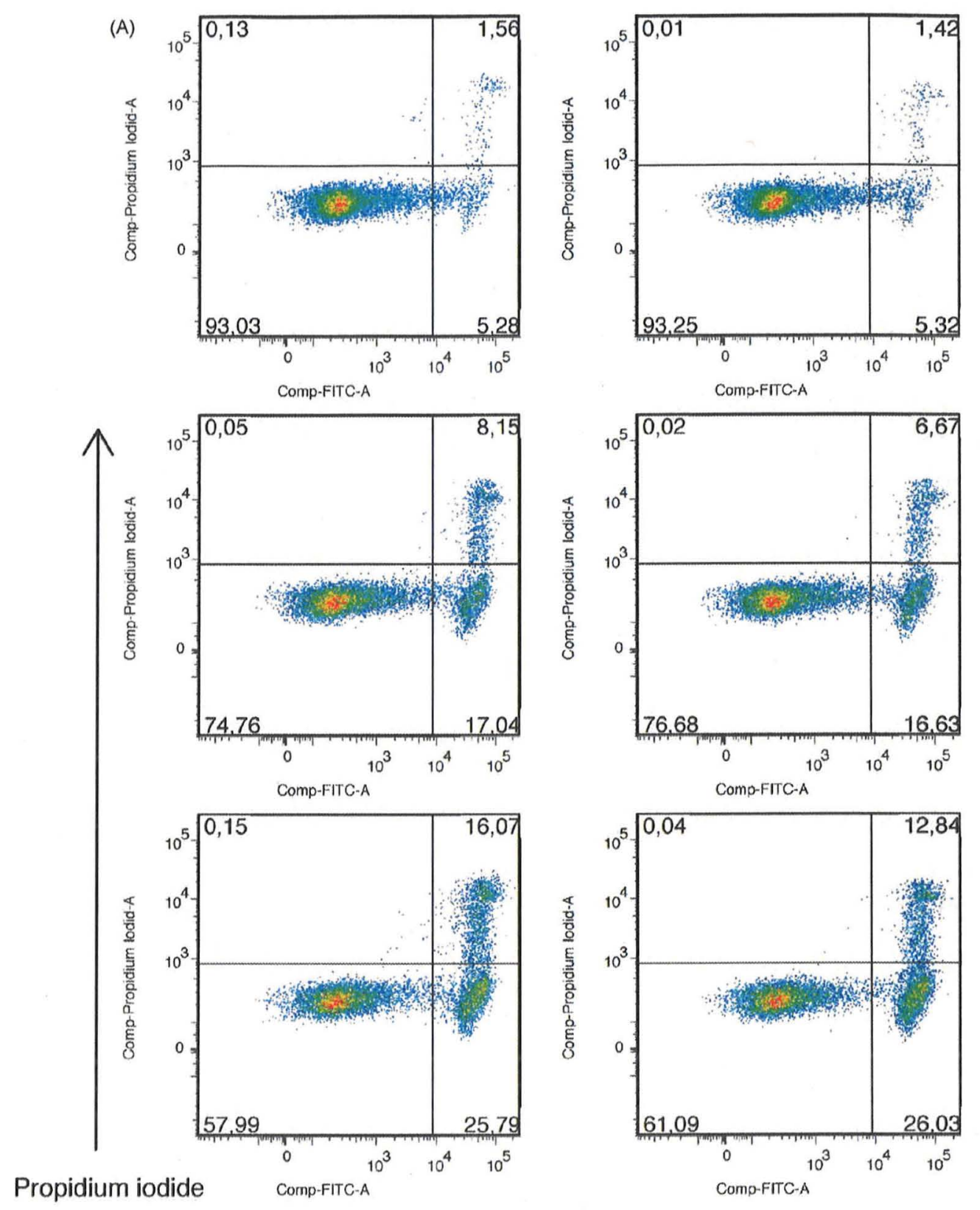

\section{Annexin V}

(B)

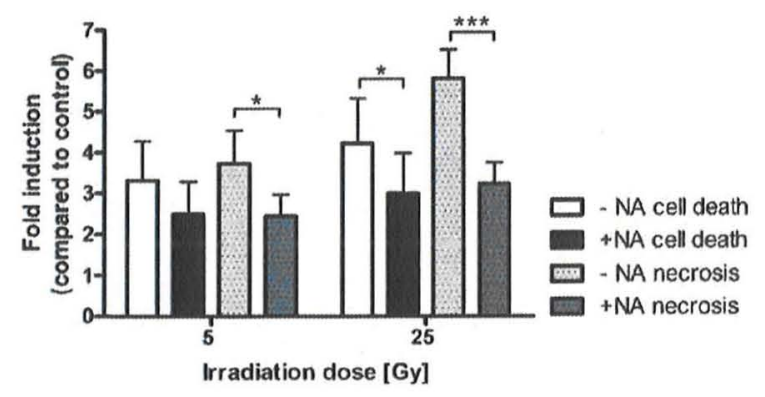

Fig. 5. Effect of NA on cell viability. NA-supplemented and non-supplemented cells were irradiated with 5 or 25 Gy, and after $24 \mathrm{~h}$ cell viability, apoptosis and necrosis was determined by flow cytometry using annexin V and propidium iodide staining. (A) Flow cytometric analysis. Viable cells (annexin V-negative/PI-negative), apoptotic cells (annexin V-positive) and cells in the necrotic stage (annexin V-positive/PI-positive) were expressed as percent. Data are representative of one out of tree measurements of one donor. (B) Comparison of the fold induction of total cell death (apoptotic and necrotic cells) between non-supplemented and supplemented cells. A significant difference was observed in irradiated cells between non-supplemented and supplemented PBMC $\left({ }^{*} p<0.05 ;{ }^{* *} p<0.01\right.$, repeated measures ANOVA). Each bar represents the mean value \pm SEM of duplicate measurements of three different donors, respectively. 


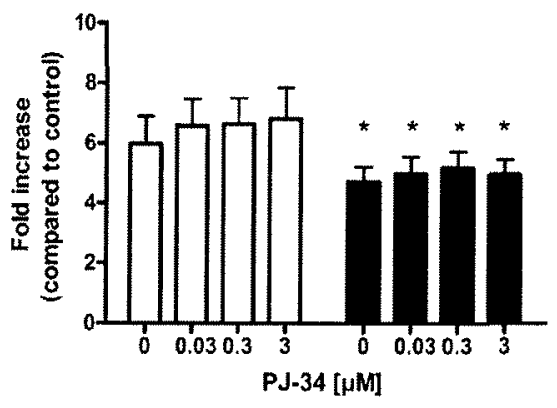

(C) Necrosis (fold increase at 5 Gy)

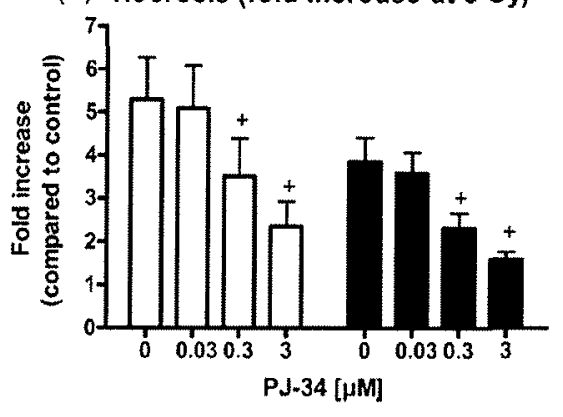

(E) Apoptosis (fold increase at $5 \mathrm{~Gy}$ )

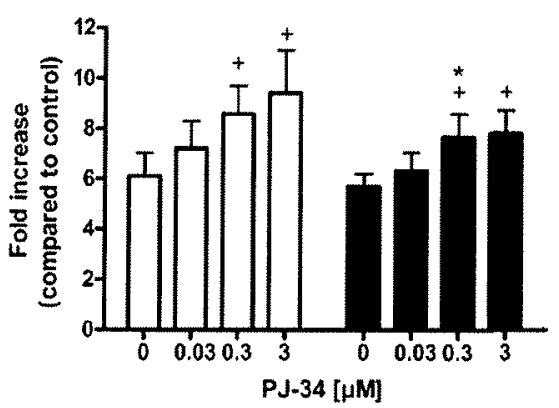

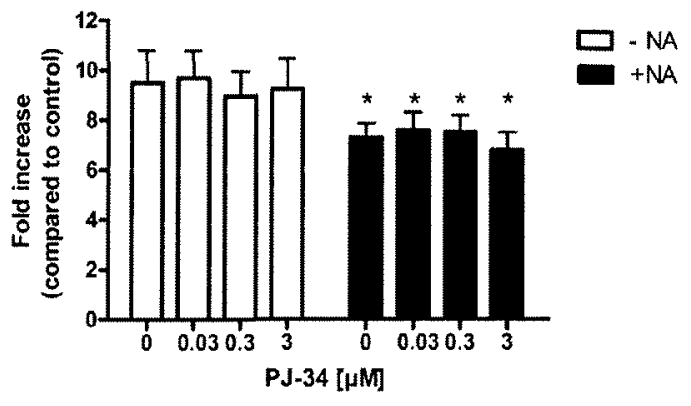

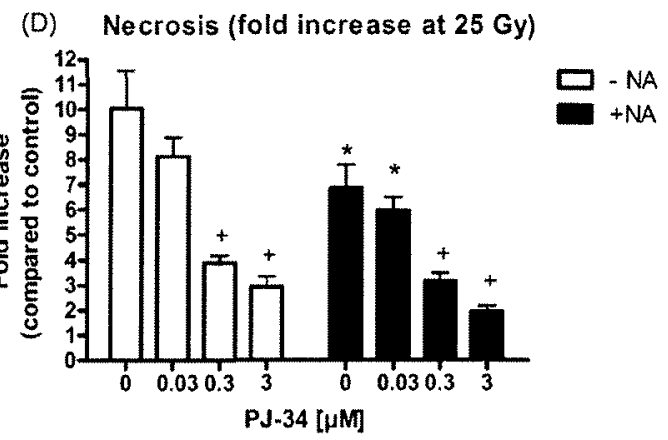

(F) Apoptosis (fold increase at $25 \mathrm{~Gy}$ )

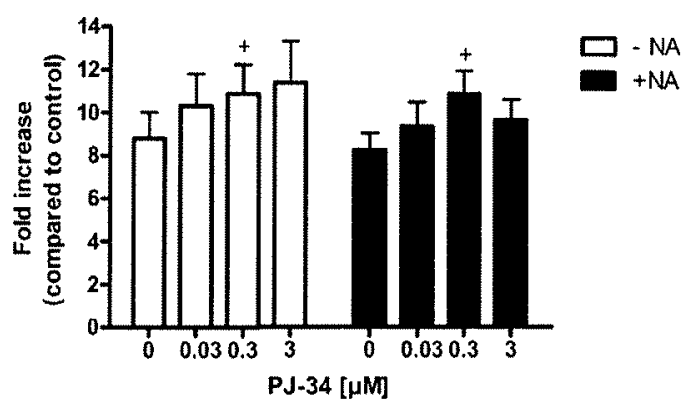

Fig. 6. Effect of PARP inhibition on cell death. PBMCs were treated with different concentrations of PJ-34 as indicated and analyzed for total cell death ( $A$ and $B$ ), necrosis ( $C$ and $D$ ) and apoptosis ( $E$ and $F$ ) after irradiation with $5 \mathrm{~Gy}(\mathrm{~A}, \mathrm{C}, \mathrm{E})$ or $25 \mathrm{~Gy}(\mathrm{~B}, \mathrm{D}, \mathrm{F})$. (A, B) A significant difference in fold-induction of total cell death was observed in irradiated cells between non-supplemented and supplemented PBMC $\left({ }^{*} p<0.05\right.$. Wilcoxon matched pairs test). (C, D, E, F) A significant decrease of the necrotic fraction with increasing concentrations of PJ-34 was detected within non-supplemented or within NA-supplemented, respectively $\left({ }^{*} p<0.05\right.$, repeated measures ANOVA), or between nonsupplemented compared to NA-supplemented ( ${ }^{*} p<0.05$, repeated measures ANOVA). Each bar represents the mean value \pm SEM of triplicate measurements of six different donors.

could extend the time span of high PARP-1 activity so that equilibrium with the catabolic activity of PARG be maintained for a longer period of time, i.e. between 1 and 7 min. Diminished PARP-1 activity can be brought about either by resealing DNA strand breaks or, if increased damage persists, by depletion of the NAD ${ }^{+}$ pool. Thereafter, degradation of the polymer can be observed. In the non-supplemented cells it is likely that the restricted NAD ${ }^{+}$ content is not able to maintain PARP-1 in a fully active state. Thus, the activity of PARG becomes dominant, resulting in rapid degradation of PAR. If the polymer is degraded before the repair process can start properly, the DNA repair process will fail, leading to mutations or apoptosis.

There are few studies in which the effect of NA on poly(ADPribosyl)ation and the cellular consequences were investigated; most of them, however, refer to correction of established NAD deficiency $[20,21]$. Nevertheless, those studies showed that PARP activity is negatively influenced by decreased cellular $\mathrm{NAD}^{+}$ concentration. In an in vivo study in rats, it was shown that supplementation of NA increases the $\mathrm{NAD}^{+}$content as well as poly(ADP-ribosyl)ation in bone marrow cells when treated with the alkylating agent ethylnitrosurea (ENU) [22]. The authors investigated the effect of NA on PAR levels $3 \mathrm{~h}$ post-treatment, when ENU starts to induce DNA damage, measured by western blot technique. However in the present study we investigated the immediate response of PARP-1 to DNA damage with the addition of cell viability analysis as readout. As overactivation of PARP-1 caused by severe DNA damage leads to depletion of $\mathrm{NAD}^{+}$, and consequently of ATP, which results in cellular dysfunction, necrosis and inflammatory response of tissue, we investigated if PBMC can be rescued from energy-loss dependent cell death by supplementation with NA. Indeed we observed increased cell viability in NAsupplemented cells after exposure to ionizing radiation (Fig. 5), which was caused by a $50 \%$ reduction in the fraction of necrotic cells. It may also be that the higher $\mathrm{NAD}^{+}$content influences other pathways such as sirtuin function, leading to higher cell viability $[38,39]$. In order to test if this effect was a direct consequence of the 
increased PARP-1 or only correlated to it, we inhibited PARP activity with the potent competitive inhibitor PJ-34. The rates of necrotic cell death of NA-supplemented and non-supplemented PBMCs were comparable at low levels when PARP-1 activity was fully inhibited by high inhibitor concentrations, but significantly different in the absence or at low concentrations of PJ-34 (Fig. 6D). This was also reflected in the levels of PAR formation detected in response to DNA damage (Fig. $4 \mathrm{~B}$ ). We propose that preserved $\mathrm{NAD}^{+}$levels even under circumstances of high PARP-1 activity after DNA damage is responsible for prevention of necrotic cell death. The increased $\mathrm{NAD}^{+}$content allows for higher PARP- 1 activity, which possibly supports DNA repair by avoiding the depletion of the $\mathrm{NAD}^{+}$pool and the subsequent cellular energy failure. Our data are in line with other studies, showing that early inhibition of PARP activity can induce a switch in the mechanism of cell death in lymphocytes [40] Moreover, we could show that combined incubation of NAD precursor NA and PARP inhibitor PJ-34 leads to an additive effect pushing cells from pro-inflammatory necrosis to less inflammatory apoptosis, which may be therapeutically useful.

In conclusion, our data show that keeping the $\mathrm{NAD}^{+}$pool high by supplementation of $N A$ leads to significantly increased poly(ADP-ribosyl)ation in response to DNA damage, even at doses where $\mathrm{NAD}^{+}$is not a limiting factor for the reaction. This results in preservation of the $\mathrm{NAD}^{+}$pool and decreases potentially harmful necrosis, comparable with the effect of PARP inhibitors. Interestingly, PAR-mediated release of apoptosis-inducing factor (AIF) from mitochondria, which leads to caspase-independent cell death [15], is not a dominant mechanism in this system, as the excessive PAR formation by nicotinic acid supplementation is not associated with increased cell death, but, on the contrary, with increased cell survival. Therapies that augment $\mathrm{NAD}^{+}$pools may be alternative pharmacological approaches to treat diseases, where PARP is pathophysiologically implicated. The consequences related with PARP-mediated reactions analyzed in this study concentrate on cell death, but the effect on DNA repair is barely understood. Further studies will address the underlying mechanisms.

\section{Acknowledgements}

This work was supported by the Deutsche Forschungsgemeinschaft through the International Research Training Group 1331 (fellowships awarded to K.W. and A.K.). The authors thank Professors M. Miwa and T. Sugimura (Tokyo, Japan) for the kind gift of $10 \mathrm{H}$ hybridoma cells. We thank Professors A. Wendel and C.R. Hauck (University of Konstanz) for kindly giving us access to their flow-cytometers.

\section{Appendix A. Supplementary data}

Supplementary data associated with this article can be found, in the online version, at doi:10.1016/j.bcp.2010.06.010.

\section{References}

[1] Benjamin RC, Gill DM. ADP-ribosylation in mammalian cell ghosts. Dependence of poly(ADP-ribose) synthesis on strand breakage in DNA. J Biol Chem 1980;255:10493-501,

[2] Amé JC. Spenlehauer $C$. de Murcia G. The PARP superfamily. Bioessays $2004 ; 26: 882-93$

[3] Otto H, Reche PA, Bazan F, Dittmar K, Haag F, Koch-Nolte F. In silico characteri$z a t i o n$ of the family of PARP-like poly(ADP-ribosyl)transferases (pARTs). BMC Genomics 2005:6:139

[4] Shieh WM, Ame JC, Wilson MV, Wang ZQ Koh DW, Jacobson MK, et al. Poly(ADP-ribose) polymerase null mouse cells synthesize ADP-ribose polymers. J Biol Chem 1998;273:30069-72

(5) Satoh MS, Lindah! T. Role of poly(ADP-ribose) formation in DNA repair. Nature 1992;356:356-8.
[6] Meyer-Ficca ML, Meyer RG, Jacobson EL, Jacobson MK, Poly(ADP-ribose) polymerases: managing genome stability. Int J Biochem Cell Biol 2005:37:920-6

[7] Gradwohl G. Ménissier de Murcia J, Molinete M, Simonin F. Koken M, Hoeijmakers JH, et al. The second zinc-finger domain of poly(ADP-ribose) polymerase determines specificity for single-stranded breaks in DNA. Proc Natl Acad Sci USA 1990:87:2990 4

[8] Ogata N, Ueda K, Kawaichi M, Hayaishi O. Poly(ADP-ribose) synthetase, main acceptor of poly(ADP-ribose) in isolated nuclei. J Biol Chem 1981; 256:4135-7.

[9] Caldecott KW, Aoufouchi S, Johnson P. Shall S. XRCC1 polypeptide interacts with DNA polymerase beta and possibly poly(ADP-ribose) polymerase, and DNA ligase $\mathrm{HI}$ is a novel molecular 'nick-sensor' in vitro. Nucleic Acids Res $1996 ; 24: 4387-94$

110] Leppard JB, Dong Z, Mackey ZB, Tomkinson AE. Physical and functional interaction between DNA ligase IIlalpha and poly(ADP-Ribose) polymerase 1 in DNA single-strand break repair. Mol Cell Biol 2003;23:5919-27.

[11] Zahradka P. Ebisuzaki K. A shuttle mechanism for DNA-protein interactions The regulation of poly(ADP-ribose) polymerase. Eur I Biochem 1982;127 579 85 .

[12] Ikejima M, Gill DM. Poly(ADP-ribose) degradation by glycohydrolase starts with an endonucleolytic incision. ] Biol Chem 1988;263:11037-40.

[13] Miwa M. Tanaka M, Matsushima T, Sugimura T. Purification and properties of glycolydrolase from calf thymus splitting ribose-ribose linkages of poly(adenosine diphosphate ribose). J Biol Chem 1974;249:3475-82

[14] Berger NA, Sims IL, Catino DM, Berger S]. Poly(ADP-ribose) polymerase mediates the suicide response to massive DNA damage: studies in normal and DNA-repair defective cells. Princess Takamatsu Symp 1983:13: and DNA-

[15] Yu SW, Wang H, Poitras MF, Coombs C, Bowers W], Federoff H], et al. Mediation of poly(ADP-ribose) polymerase-1-dependent cell deatl by apoptosis-inducing factor. Science 2002;297:259-63.

[16] Rongvaux A, Andris F, Van Gool F, Leo O. Reconstructing eukaryotic NAD metabolism. Bioessays 2003:25:683-90.

[17] Bieganowski P. Brenner $C$. Discoveries of nicotinamide tiboside as a nutrient and conserved NRK genes establish a Preiss-Handler independent route to $\mathrm{NAD+}$ in fungi and humans. Cell 2004;117:495-502.

[18] Jackson TM, Rawling JM, Roebuck BD, Kirkland JB. Large supplements of nicotinic acid and nicotinamide increase tissue NAD+ and poly(ADP-ribose) levels but do not affect diethylnitrosamine-induced altered hepatic foci in Fischer-344 rats. J Nutr 1995;125:1455-61.

[19] Durkacz BW, Omidiji O, Gray DA, Shall S. (ADP-ribose)n participates in DNA excision repair. Nature 1980;283:593-6.

[20] Boyonoski AC, Spronck JC, Gallacher LM, Jacobs RM, Shah GM, Poirier GG, et al. Niacin deficiency decreases bone marrow poly(ADP-ribose) and the latency of ethylnitrosourea-induced carcinogenesis in rats. J Nutr 2002 ; 132:108-14.

[21] Rawling JM, Jackson TM, Driscoll ER, Kirkland JB. Dietary niacin deficiency lowers tissue poly(ADP-ribose) and NAD+concentrations in Fischer-344 rats. I Nutr 1994:124:1597-603.

[22] Boyonoski AC, Spronck JC, Jacobs RM, Shah GM, Poirier GG, Kirkland JB. Pharmacological intakes of niacin increase bone marrow poly(ADP-ribose) and the latency of ethylnitrosourea-induced carcinogenesis in rats. J Nutr 2002:132:115-20.

[23] Kurnick JT, Ostberg L, Stegagno M. Kimura AK, Orn A. Sjoberg O. A rapid method for the separation of functional lymphoid cell populations of human and animal origin on PVP-silica (Percoll) density gradients. Scand J Immuno $1979 ; 10: 563-73$

[24] Kawamitsu H, Hoshino H, Okada H, Miwa M, Monoi H, Sugimura T. Monoclonal antibodies to poly(adenosine diphosphate ribose) recognize different structures. Biochemistry 1984;23:3771-7.

[25] Jacobson EL, Jacobson MK. Pyridine nucleotide levels as a function of growth in normal and transformed 3T3 cells. Arch Biochem Biophys 1976;175 $627-34$.

[26] Jacobson EL, Dame AJ, Pyrek JS, Jacobson MK, Evaluating the role of niacin in human carcinogenesis. Biochimie 1995:77:394-8.

[27] Kirkland JB. Niacin status, NAD distribution and ADP-ribose metabolism. Curr Pharm Des 2009;15:3-11.

[28] Kunzmann $A$, Liu $D$, Annett $K$, Malaise $M$, Thaa $B$, Hyland $P$, et al. Flowcytometric assessment of cellular poly(ADP-ribosyl)ation capacity in peripheral blood lymphocytes. Immun Ageing 2006;3:8

[29] Pfeiffer R, Brabeck C, Bürkie A. Quantitative nonisotopic immuno-dot-blo method for the assessment of cellular poly(ADP-ribosyl)ation capacity. Anal Biochem 1999;275:118-22.

[30] Jacobson MK, Payne DM, Alvarez-Gonzalez R, Juarez-Salinas H. Sims JI Jacobson EL. Determination of in vivo levels of polymeric and monomeric ADP-ribose by fluorescence methods. Methods Enzymol 1984;106 483-94.

[31] Juarez-Salinas H, Sims JL, Jacobson MK. Poly(ADP-ribose) levels in carcinogentreated cells. Nature 1979:282:740-1.

[32] Brabeck $C$, Pfeiffer $R$, Leake A, Beneke S, Meyer $R$, Bürkle A. L-Selegiline potentiates the cellular poly(ADP-ribosyl)ation response to ionizing radiation J Pharmacol Exp Ther 2003;306:973-9.

(33) Küpper JH, de Murcia $G$, Bürkle A. Inhibition of poly(ADP-ribosyl)ation by overexpressing the poly(ADP-ribose) polymerase DNA-binding domain in mammalian cells. J Biol Chem 1990;265:18721 4. 
[34] Kunzmann A, Dedoussis G, Jajte ], Malavolta M, Mocchegiani E, Bürkle A. Effect of zinc on cellular poly(ADP-ribosyl)ation capacity. Exp Gerontol 2008; 43:409-14

[35] Ogata S, Okumura K, Taguchi $\mathrm{H}$. The effects of niacin on DNA repair after $\mathrm{N}$ methyl- $\mathrm{N}^{\prime}$-nitro- $\mathrm{N}$-nitrosoguanidine treatment in normal human lymphocytes. Biosci Biotechnol Biochem 1997;61:2116-8.

[36] Alvarez-Gonzalez R, Jacobson MK. Characterization of polymers of adenosine diphosphate ribose generated in vitro and in vivo. Biochemistry 1987:26:3218-24.

[37] Hatakeyama $K$, Nemoto $Y$, Ueda $K$, Hayaishi $O$. Purification and characterization of poly(ADP-ribose) glycohydrolase. Different modes of action on large and small poly(ADP-ribose). J Biol Chem 1986;261: 14902-11.

[38] Yang $T$, Sauve AA, NAD metabolism and sirtuins: metabolic regulation of protein deacetylation in stress and toxicity. AAPS J 2006;8:E632-43.

[39] Araki T, Sasaki Y, Milbrandt J. Increased nuclear NAD biosynthesis and SIRT1 activation prevent axonal degeneration. Science 2004;305: $1010-3$

[40] Meier HL, Millard CB. Alterations in human lymphocyte DNA caused by sulfur mustard can be mitigated by selective inhibitors of poly(ADP-ribose) polymerase. Biochim Biophys Acta 1998;1404:367-76. 